\title{
Factores que influyen en la inasistencia a sus terapias, en niños con discapacidad
}

\author{
Factors that influence in the absence of their therapies, in children with disabilities
}

\section{Sr. Editor:}

Las terapias constituyen los pilares de la rehabilitación en niños con discapacidad, mediante las cuales se educa al niño para el logro de una mayor independencia funcional posible y se orienta a los padres para continuar las actividades terapéuticas en casa. La rehabilitación en el caso de los niños con discapacidad presenta características que los diferencian de otros pacientes. La madre generalmente lo traslada y tiene que afrontar una serie de problemas, aparte de afrontar el tener un niño con discapacidad tiene que dar solución a lo económico, las ocupaciones de la casa, las enfermedades propias del niño, los tiempos de traslado, aspectos que muchas veces no son considerados en su atención y que son factores para la inasistencia a sus terapias. Los padres consideran difícil hacer la rutina en casa, que los niños no trabajan igual en casa o el temor de no realizar los ejercicios en casa correctamente (1).
En nuestro país no hay estudios que aborden directamente esta problemática, en otros países los estudios abordan la falta de adherencia que es la falta de cumplimiento de las indicaciones del profesional por el paciente,que muestran como causa principal de no recibir atención a la falta de dinero en 52,3\%, la lejanía del centro en 3,2\% y no hay quien lo lleve $1,6 \%$ (2). En otro estudio, la falta de adherencia llegó a $70,4 \%$ en los que viven lejos frente a $48,5 \%$ en los que viven cerca (3).

Maco MB (4), en su tesis de licenciatura, refiere que el $34 \%$ de inasistencia a terapias es por los escasos recursos económicos, afectando el bienestar del niño con discapacidad. En una investigación sobre deficiencia y discapacidad en el Instituto Nacional de Rehabilitación, se encontró que la mayoría de pacientes provenían de distritos cercanos y un grupo reducido de distritos alejados (5). Un estudio realizado en México refirió que los niños con parálisis cerebral, cuyos

Tabla 1.Distribución de la inasistencia por actividad terapéutica de pacientes niños en rehabilitación.

\begin{tabular}{|c|c|c|c|c|c|c|c|c|}
\hline \multirow{2}{*}{ Inasistencia (\%) } & \multicolumn{2}{|c|}{ Terapia física } & \multicolumn{2}{|c|}{$\begin{array}{c}\text { Terapia } \\
\text { Ocupacional }\end{array}$} & \multicolumn{2}{|c|}{ Trabajo social } & \multicolumn{2}{|c|}{ Psicología } \\
\hline & $\mathrm{n}$ & $\%$ & $\mathrm{n}$ & $\%$ & $\mathrm{n}$ & $\%$ & $\mathrm{n}$ & $\%$ \\
\hline $0-19$ & 169 & 34,6 & 121 & 39,4 & 213 & 68,9 & 157 & 53,2 \\
\hline $20-49$ & 133 & 27,3 & 75 & 24,4 & 2 & 0,6 & 1 & 0,3 \\
\hline $50-74$ & 64 & 13,1 & 53 & 17,3 & 6 & 1,9 & 2 & 0,7 \\
\hline $75-99$ & 47 & 9,6 & 25 & 8,1 & 0 & 0 & 0 & 0 \\
\hline 100 & 75 & 15,4 & 33 & 10,7 & 88 & 28,5 & 135 & 45,8 \\
\hline Total & 488 & 100 & 307 & 100 & 309 & 100 & 295 & 100 \\
\hline
\end{tabular}

Departamento de Investigación, Docencia y Rehabilitación Integral en el DesarrolloPsicomotor; Instituto Nacional de Rehabilitación 'Dra. Adriana Rebaza Flores'Amistad Perú-Japón. Lima, Perú.

Facultad de Medicina, Universidad Nacional Mayor de San Marcos. Lima, Perú.

a Especialista en Fisioterapia en Neurorrehabilitación; ${ }^{b}$ Licenciado Tecnólogo Medico, especialidad Terapia Física y Rehabilitación; ${ }^{\mathrm{c}}$ Magister en Salud Ocupacional; d Docente Principal. 
Tabla 2. Inasistencia de pacientes niños en rehabilitación según causa referida por los padres.

\begin{tabular}{|c|c|c|c|c|c|c|c|c|c|c|c|c|}
\hline \multirow{2}{*}{ Inasistencia (\%) } & \multicolumn{2}{|c|}{ Económico } & \multicolumn{2}{|c|}{ Enfermedad } & \multicolumn{2}{|c|}{$\begin{array}{l}\text { Trabajo de } \\
\text { padres }\end{array}$} & \multicolumn{2}{|c|}{ Otros } & \multicolumn{2}{|c|}{ No especifica } & \multicolumn{2}{|c|}{ Total } \\
\hline & $\mathrm{n}$ & $\%$ & $\mathrm{n}$ & $\%$ & $\mathrm{n}$ & $\%$ & $\mathrm{n}$ & $\%$ & $\mathrm{n}$ & $\%$ & $\mathrm{n}$ & $\%$ \\
\hline \multicolumn{13}{|l|}{ Terapia física } \\
\hline $20-49$ & 3 & 16,7 & 3 & 16,7 & 1 & 5,6 & 3 & 16,7 & 8 & 44,4 & 18 & 100 \\
\hline $50-74$ & 3 & 50,0 & 1 & 16,7 & 0 & 0 & 2 & 33,3 & 0 & 0 & 6 & 100 \\
\hline $75-99$ & 0 & 0 & 3 & 42,9 & 1 & 14,3 & 2 & 28,6 & 1 & 14,3 & 7 & 100 \\
\hline 100 & 1 & 20,0 & 0 & 0 & 1 & 20,0 & 1 & 20,0 & 2 & 40,0 & 5 & 100 \\
\hline Total & 7 & 19,4 & 7 & 19,4 & 3 & 8,3 & 8 & 22,2 & 11 & 30,6 & 36 & 100 \\
\hline \multicolumn{13}{|l|}{ Terapia ocupacional } \\
\hline $20-49$ & 2 & 16,7 & 2 & 16,7 & 1 & 8,3 & 2 & 16,7 & 5 & 41,7 & 12 & 100 \\
\hline $50-74$ & 1 & 14,3 & 1 & 14,3 & 0 & 0 & 0 & 0 & 5 & 71,4 & 7 & 100 \\
\hline $75-99$ & 0 & 0 & 1 & 33,3 & 1 & 33,3 & 1 & 33,3 & 0 & 0 & 3 & 100 \\
\hline 100 & 4 & 57,1 & 0 & 0 & 0 & 0 & 3 & 42,9 & 0 & 0 & 7 & 100 \\
\hline Total & 7 & 24,1 & 4 & 13,8 & 2 & 6,9 & 6 & 20,7 & 10 & 34,5 & 29 & 100 \\
\hline
\end{tabular}

padres evidenciaban depresión grave, presentaron elevado número de inasistencia a terapias (6).

En el Instituto Nacional de Rehabilitación cuando estaba ubicado en el Callao en 1998, se realizó un estudio en el Departamento de Desarrollo Psicomotor (estudio no publicado), con el objetivo de determinar los motivos de inasistencia a terapias de los niños con discapacidad que asistían para su rehabilitación. Comprendió dos etapas, en la primera se seleccionaron las historias de los pacientes atendidos con indicación para todas las actividades terapéuticas en un año. Los mayores porcentajes cuando el paciente era programado y no asistía a ninguna de sus citas (total),fueron en Psicología con 45,8\% y Trabajo social $28,5 \%$ (Tabla 1 ).

En la segunda fase se seleccionó el $15 \%$ de las historias clínicas y se realizó una encuesta a los padres de los pacientes en su domicilio. En los pacientes que presentaron $20 \%$ a mas de inasistencia, tanto para la terapia física y la terapia ocupacional el motivo principal que refirieron los padres fue el económico y la enfermedad (Tabla 2).

Es necesario realizar investigaciones sobre los factores que influyen en la inasistencia a terapias y así establecer medidas que permitan una intervención oportuna y la continuidad, considerando que un niño está en desarrollo y lo que no logra a temprana edad se hace más difícil rehabilitar en una edad mayor donde las limitaciones pueden haberse establecido.

\section{Herminio Teófilo Camacho-Conchucos ${ }^{1, a, b ; ~ 2, b, c ~}$}

\section{Correspondencia:}

Herminio Teófilo Camacho Conchucos

Dirección: Calle Ícaro 105 Urb. La Campiña,

Chorrillos. Lima, Perú.

Teléfono: (511) 4672103 / (51) 999251213.

Correo electrónico: teocamacho20@hotmail.com

\section{REFERENCIAS BIBLIOGRÁFICAS}

1. Rossini Y, Estrada OL, Tejada PA, Crespo O. Factores relacionados con adherencia a un programa de rehabilitación. Revista Facultad de Salud. 2010; 2(1):39-50. (Citado el 2 de enero del 2018); Disponible en: https://www.journalusco. edu.co/index.php/rfs/article/view/28/30

2. Tapias LM. Factores individuales que influyen en la adherencia al tratamiento fisioterapéutico. Una revisión del tema. Rev CES Mov y Salud. 2014; 22(1):23-30. (Citado el 4 de enero del 2018) Disponible en: http://revistas.ces.edu.co/index. php/movimientoysalud/article/view/2969/pdf

3. Coello-Talavera D, Rojas-Motta N, Mayta-Tristan P. Falta de adherencia al tratamiento fisioterapéutico en pacientes con trastornos musculoesqueléticos 
en una clínica docente en Lima, Perú. Fisioterapia. 2016;38(4):182-8.

4. Maco MB. Factores familiares que limitan el bienestar físico y emocional de los niños con discapacidad del hospital Belén, provincia de Trujillo, región La Libertad, en el año 2013. Tesis de Licenciatura. Trujillo, Perú: Universidad Nacional de Trujillo; 2014. 141 pp.

5. Camacho-Conchucos HT, Fajardo-Campos P, Zavaleta de Flores E. Análisis descriptivo sobre deficiencias y discapacidades del desarrollo psicomotor en pacientes atendidos en el Instituto Nacional de Rehabilitación 2006-2008. An Fac Med. 2012;73(2):119-26.

6. Rubio R, Gutiérrez MDC, Castellanos Valencia A, Flores EM. Salud mental en cuidadores primarios de niños con parálisis cerebral en rehabilitación. Rev Psicoter. 2015;26(102):133-44.

Recibido: 10/02/2018 